\title{
Pulsatile weight increases in very low birthweight babies appropriate for gestational age
}

\author{
L Greco, A Capasso, C De Fusco, R Paludetto
}

\begin{abstract}
The weight increment profiles of 20 low birthweight babies measured during the first two months of extrauterine life were analysed. The babies were weighed daily, and the weight profiles showed minor irregularities when compared with an interpolated linear trend. When increments were plotted at two week intervals a linear increase in weight velocity was seen, but when increments were computed every three days, the velocity profile was non-linear and pulsatile. All cases studied showed regular pulsatile patterns of weight velocity during the first two months of life. A mean profile of the 20 babies permitted estimation of the periodicity of the pulsing: the cycle alternated every nine to 11 days. A non-linear pattern was found in the published series of unsmoothed data that have been widely adopted as standards for growth in low birthweight babies.
\end{abstract}

Survival of low birthweight infants is strictly related to the birth weight and to the weight increments after birth. The aim of this paper was to evaluate the adequacy of the growth centile charts in common use, most of which were derived from cross sectional data. ${ }^{1-7}$

Many of the available centile charts were constructed by pooling data from different centres, as low birthweight babies are comparatively rare. The actual drawing of the centile lines through the mean values (and the points derived by mean (SD) units) were calculated by graphic, ${ }^{3}$ as well as mathematical smoothing (three point means, for example, in the charts of Lubchenco et $a l^{4}$ ). Most data points are derived by linear extrapolation from raw data points, with an assumption of linearity between one time and the next; regression methods were often used. ${ }^{137}$

Few longitudinal studies have been carried out, and data from individual subjects are rare, as all published reports deal with pools or group means. The velocity, or the dynamic growth of the individual low birthweight infant is a good monitor of his well being, and is carefully monitored in each nursery, but is rarely published. A velocity curve may be helpful to monitor the adequacy of the increments of the individual subject. The object of this paper was to analyse the individual patterns of weight increase of a small cohort of low birthweight babies who were weighed accurately every day. Length measurements were not considered because of the potential for errors of measurement.
Subjects and methods

Twenty low birthweight babies were weighed nude before feeding every day on a Vandoni scale accurate to the nearest $5 \mathrm{~g}$ by experienced nurses. The mean (SD) difference in measurements (obtained by 64 duplicate measurements) was $12 \cdot 2(3 \cdot 4) \mathrm{g}$.

Eleven girls and nine boys were born between 26 and 30 weeks' gestation with birth weights ranging from 930 to $2000 \mathrm{~g}$. Most had the common complaints of preterm babies: six required assisted ventilation, 15 were given blood transfusions, seven had apnoeic episodes, five had severe jaundice, and 11 had sepsis.

Velocities were expressed in $\mathrm{g} /$ day, and were calculated by dividing the increments between two intervals by the days, and plotting the mid interval between measurements.

\section{Results}

Figure 1 shows the weight profile of a baby born

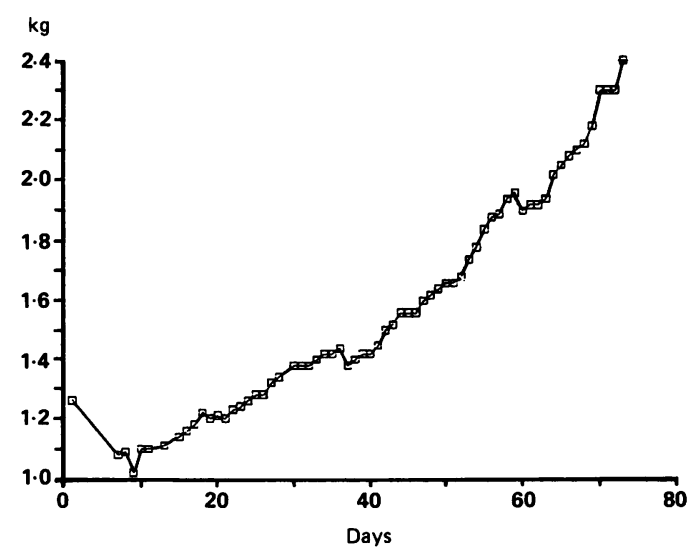

Figure 1 Weight profile of case 1.

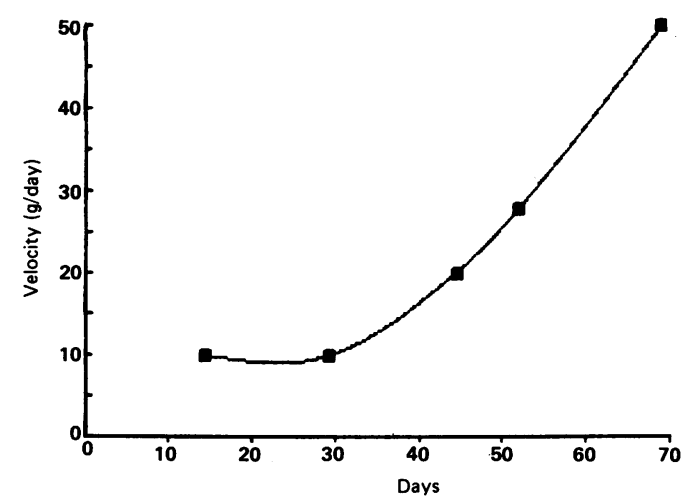

Figure 2 Weight velocity profile obtained by computing increments at 15 day intervals (case 1). 
Figure 3 Weight velocity profile obtained by computing increments at 15 and

10 day intervals (case 1 ). The triangles indicate 15 day measurements and the squares 10 day measurements.
Figure 4 Weight velocity profile obtained by computing increments at three day intervals (case 1).
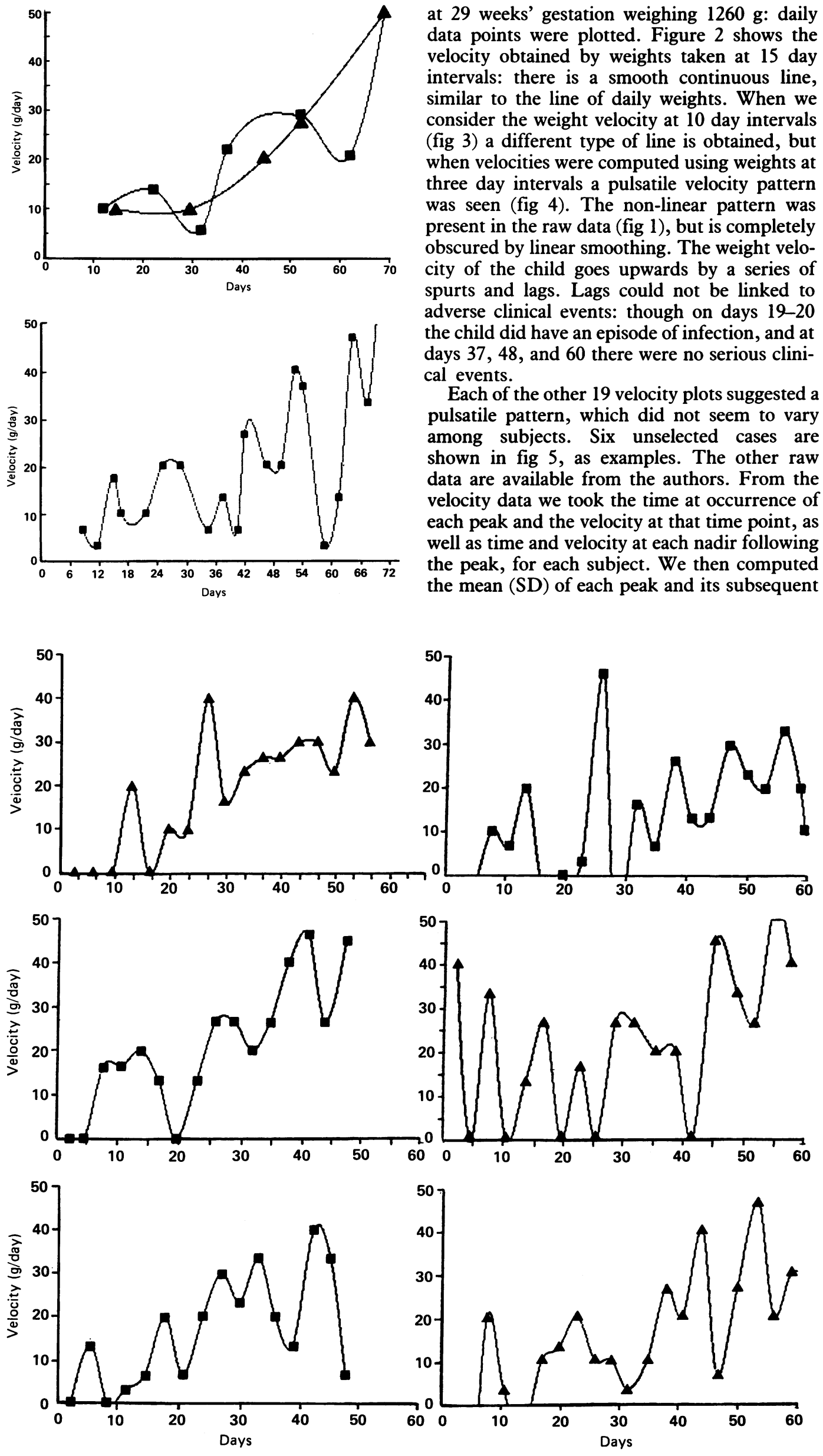

at 29 weeks' gestation weighing $1260 \mathrm{~g}$ : daily data points were plotted. Figure 2 shows the velocity obtained by weights taken at 15 day intervals: there is a smooth continuous line, similar to the line of daily weights. When we consider the weight velocity at 10 day intervals (fig 3) a different type of line is obtained, but when velocities were computed using weights at three day intervals a pulsatile velocity pattern was seen (fig 4). The non-linear pattern was present in the raw data (fig 1), but is completely obscured by linear smoothing. The weight velocity of the child goes upwards by a series of spurts and lags. Lags could not be linked to adverse clinical events: though on days 19-20 the child did have an episode of infection, and at days 37,48 , and 60 there were no serious clinical events.

Each of the other 19 velocity plots suggested a pulsatile pattern, which did not seem to vary among subjects. Six unselected cases are shown in fig 5, as examples. The other raw data are available from the authors. From the velocity data we took the time at occurrence of each peak and the velocity at that time point, as well as time and velocity at each nadir following the peak, for each subject. We then computed the mean (SD) of each peak and its subsequent (1)

Figure 5 Three day weight velocity profiles of six low birthweight babies. 


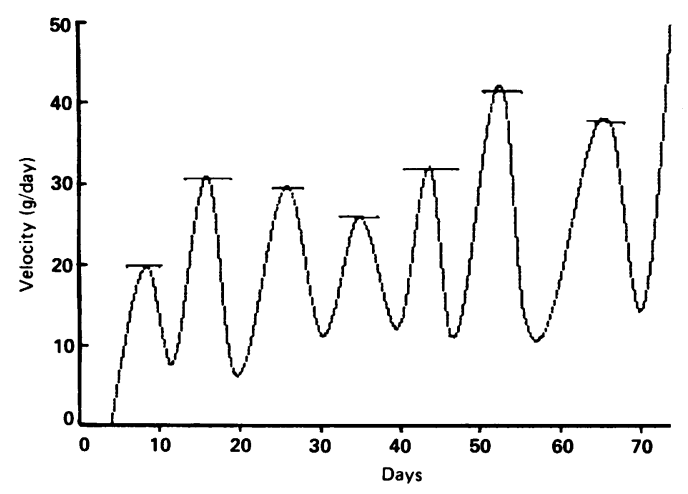

Figure 6. Profile of the mean weight velocity pattern of 20 low birthweight babies. Horizontal lines indicate $1 S D$ intervals of the peaks.

nadir. No smoothing was applied to the data points and no assumption about an underlying mathematical model was made.

Figure 6 shows the pattern obtained by the plot of these mean values. A constant pulsatile pattern is suggested by the graph, with a distance from peak to peak of eight to 11 days (mean (SD) $9 \cdot 55(2 \cdot 18)$ days).

\section{Discussion}

Low birthweight babies increase their weight by a pulsatile rhythm, the period between peaks being about 10 days. Their weight increase is not linear as previously reported; the linearity is only the result of the extensive use of graphic as well as of mathematical smoothing. The currently available centiles charts are useful in clinical practice, in that they allow comparison of an individual with a group mean, but they may not be adequate for the plot of longitudinal data of a single subject. New studies are therefore required, as well as a re-evaluation of the longitudinal data already available.

The lack of velocity data in the neonatal period may be because of the inaccuracy of the measurements. To get firm velocity data the longest period between two measurements must be taken, as short time growth evaluation may be misinterpreted because of such inaccuracies of measurement. As far as weight increments are concerned, most reports give an error of measurement of about $10 \mathrm{~g} .{ }^{137}$ For example, a low birthweight infant will gain an average of $300 \mathrm{~g}$ between the 32nd and the 33rd week, which is 30 times larger than the error of measurement.

Velocity data may therefore be interpreted with adequate control of the error of measurement, at least as far as weight is concerned. The general assumption about the growth of low birthweight infants is that it is linear, which is the philosophy underlying the concept of the centile graph in low birthweight babies as well as in those born at full term of normal birth weight. ${ }^{134}$ Specific statements have ruled out the possibility of a growth process that is not continuous and linear. ${ }^{8}$ Nevertheless, it is generally accepted that a baby born with a low birth weight before full term has to go through some variation in growth velocity, as he has to go through a period of negative velocity, a period of catch up growth, and a period of stable velocity. Gairdner and Pearson described 'one of the commoner patterns of weight gains to be seen in prematures and small for date infants' in four 'phases': the first of weight loss, the second (after the first week) parallel to a fetal weight curve, the third of growth acceleration and catch up, and the fourth of stability along the individual centile. ${ }^{2}$

Brandt reported accurate individual growth data of a cohort of 64 infants whose weights were appropriate for their gestational age and 43 who were small for their gestational ages, with weekly, fortnightly, and monthly measuring intervals. The velocity curves were drawn from the means of the velocity of each individual, but they still showed some irregularities, possibly because of the multiple peaks that indicated the height as well as the weight values. ${ }^{7}$ If we consider the widely used reference data of Lubchenco et $a l,{ }^{4}$ the plot of the velocities obtained by points interpolated on the smoothed 50th centile line give a single peak of maximum velocity around the 34th week of gestation, but if we compute the increments between the pooled mean weights given for boys and girls in the same paper, the pattern is that of a pulsatile growth, although cross sectional pooled data of both sexes with a comparatively wide interval between measurements are considered.

The suggested pulsatile pattern of growth is not unique to the weight of low birthweight babies: most probably it is a universal pattern of the physiology of growth. It may be found in the growth of primates, ${ }^{8}$ and in almost every human measurement in which it has been sought. Healthy subjects measured longitudinally over a long period and with great accuracy showed a variation with time, with 'mini spurts' alternating with lag phases during the whole growth period in weight, height, urinary urea concentration, and creatinine excretion. ${ }^{9} 10$ The application of high precision knemometry to a wide variety of subjects, both healthy and affected by growth disorders, showed a pulsatile pattern in the growth of the leg in all the subjects studied. ${ }^{11}$

Children with coeliac disease showed a pattern of pulsatile growth in height as well as in weight during the catch up growth that followed the introduction of the gluten free diet; the cycle showed a periodicity of about two months, and during that period the growth velocity was two to four times that expected for the age of the child. ${ }^{12}$

The mathematical models recently developed to fit the human growth curve are adequate for describing the general trend of the growth profile, but new time series models have to be developed to fit the velocity variations of short term growth adequately.

1 De Curtis M, Bland JM, Paone MC, Vetrano G, Paludetto R, Ciccimarra F. Birth weight of Italian infants of 30 weeks gestation or less. Eur $\mathcal{F}$ Pediatr 1988;148:136-8.

2 Gairdner D, Pearson J. A growth chart for premature and other infants Arch Dis Child 1971;46:783-7.

3 Babson SG, Benda GI. Growth graphs for the clinical assessment of infants of varying gestational age $\mathcal{f}$ Pediatr 1976; 89:814-20. 
4 Lubchenco LO, Hansman C, Dressler M, Boyd E. Intrauterine growth as estimated from liveborn birth-weight data at 24 to 42 weeks of gestation. Pediatrics 1963;32: 793-800

5 Brooke OG, McIntosh N. Birthweights of infants born before 30 weeks' gestation. Arch Dis Child 1984;59:1189-90.

6 Karlberg P, Niklasson A, Ericson A, et al. A methodology for evaluating size at birth. Acta Paediatr Scand 1985; 319(suppl):26-37.

7 Brandt I. Growth dynamics of low-birth-weight infants with emphasis on the perinatal period. In: Falkner $F$, Tanner JM, eds. Human growth. London: Plenum Press, 1978:557-617.

8 Tanner JM. Foetus into man. London: Open Books, 1978.

9 Togo M, Togo T. Initiation time of adolescent growth spurt estimated by a certain trough in time-series analysis of monthly anthropometric and urinalysis data in five siblings. Hum Biol 1988;60:223-35.

10 Butler GE, Ratcliffe SG. An analysis of the phases of midchildhood growth by synchronization of the growth spurts. In: Tanner JM, ed. Auxology 88: perspectives in the science of growth and development. London: Smith-Gordon, 1989: growth

11 Hermanussen $M$. Knemometry, a new tool for the investiga-

tion of growth. Eur $\mathcal{f}$ Pediatr 1988;147:350-5.
12 Greco L, Tozzi AE, Capozzi G, Mayer M, Grimaldi M. Catch up growth in coeliac children: longitudinal growth study of 300 cases. In: Tanner JM, ed. Auxology 88: perspectives in the science of growth and development. London: Smiththe science of growth
Gordon, 1989:319-23. 